\title{
Gerencia y Sociedad del Conocimiento para Optimizar el Desempeño Académico del Docente
}

\author{
Autora: María Mercedes Carrillo \\ Universidad Nacional Experimental "Rafael María Baralt", UNERMB \\ mercecar-42-68@hotmail.com \\ Trujillo, Venezuela
}

\section{Resumen}

La sociedad experimenta transformaciones aceleradas, producto de las olas de cambios con el devenir de los años. Cada día plantea nuevos desafíos a los gerentes de las organizaciones. De ahí que, el presente artículo tiene como objetivo "Identificar la importancia de la Gerencia y Sociedad del Conocimiento para Optimizar el Desempeño Académico del Docente". Metodológicamente, el artículo se sustenta en una investigación de tipo documental-descriptiva, sustentada en la lectura de reconocidos autores; textos bibliográficos para dar soporte al análisis teórico-bibliográfico. Como conclusiones, se tienen: La nueva gerencia se enfrenta a un cambio de aprendizaje; la sociedad refleja información, conocimiento; Estamos ante un paisaje de retos, como la creación del conocimiento; La educación representa un factor crucial en esta transformación social. Finalmente, analizando los resultados se pudo evidenciar en la temática tratada que los textos consultados y aportes de los teóricos indagados dieron sustento y relevancia científica al artículo presentado.

Palabras clave: gerencia; conocimiento; sociedad del conocimiento; desempeño académico del docente. 


\title{
Knowledge Management Society to Optimize Teaching Academic Performance
}

\begin{abstract}
Society is undergoing rapid changes as a result of the waves of change with the passing of the years. Each day brings new challenges to managers of organizations. Hence, this paper aims to "identify the importance of Management and Knowledge Society to Optimize Teaching Academic Performance". Methodologically article is based on an investigation of documentary-descriptive, based on recognized authors reading; bibliographic texts to support the theoretical literature review. In conclusion, there are: The new management faces a change of learning; It reflects information society, knowledge; We are facing a landscape of challenges, such as the creation of knowledge; Education is a crucial factor in this social transformation. Finally, analyzing the results was evident in the treated subject that the texts consulted and contributions of investigated theoretical gave support and scientific relevance article presented.
\end{abstract}

Keywords: management; knowledge; knowledge society; academic teacher performance. 


\section{Introducción}

La noción de posmodernidad es un ciclo histórico caracterizado por un modo de pensar diferente, discípulo del desencanto y susceptibilidad en la razón ilustrada, dada por el carácter autónomo que le atribuye la vigencia de leyes impuestas para el entendimiento y razón, propio de la época de modernidad. De este modo, se da por la potestad de lo operativo del conocer, es acomodar el mundo a través de los conceptos, mientras la razón ejerce funciones regulativas del deducir la conciencia piensa, mientras el entendimiento opera con concepciones y fenómenos. De allí que, la condición postmoderna cuestiona la visión única del mundo moderno.

Desde esta premisa, el surgimiento de la postmodernidad, está asociado a cambios, descubrimientos y crisis sociales del siglo $\mathrm{XX}$, al igual con el desarrollo de la informática, telemática, que han sucedido en todos los campos de la sociedad. Las ideas de los postmodernos, es exponer un convenio en tanto que, razones cognoscentes y atrevimientos libres son obtenidos por medio del dialogo. Así, especifican en valorar todas las racionalidades que se den en el ser humano, admitiendo las interpretaciones, sin despreciar las contradicciones.

Sobre la base de estas expresiones, el actual período histórico de la postmodernidad del siglo XXI, está ofreciendo un nuevo escenario que transforma el producto de la actividad intelectual en capital social, otorgando a la educación el compromiso de sembrar aptitudes y esencialmente la capacidad de aprender a aprender como centro de todo proyecto educativo, porque "aprender" es una capacidad necesaria y humana, es la esencial de la sociedad del conocimiento.

Desde estas ideas, el asiento de la postmodernidad se conforma y desenvuelve en una sociedad susurrada por el conocimiento, aludiendo las organizaciones posmodernas al respecto Useche y Queipo (2005): exponen "requieren que sus principales actores enfrenten a viejos temores, 
contradicciones, tabúes y hacia una transformación por su propia naturaleza, lo cual no es estacionaria, debe crear continuamente nuevos elementos como la electrónica, la informática, la robótica, y la biotecnológica" es decir, que el talento humano sea el principal (pág. 65).

Amparados en esta visión, la posmodernidad reconoce la calidad que tiene en estos tiempos de permutaciones, la visión del recurso humano, como actor creador e innovador, y la reinversión organizacional para poder ajustar, superarse a sí misma, relevando procesos, productos y servicios. Atendiendo al orden precedente, la Organización de las Naciones Unidas para la Ciencia, la Educación y la Cultura, UNESCO (2009) en particular, ha adoptado el término "sociedad del conocimiento", o su variante, "sociedades del saber", dentro de sus políticas institucionales (pág. 2). Por esto, ha desarrollado una reflexión en torno al tema, buscando incorporar una concepción más integral, no ligada solamente a la dimensión económica, donde se hace mención a los avances tecnológicos circundantes en los espacios sociales.

En este orden de ideas, la tarea básica de la gerencia es encauzar actividades con la participación de las personas, porque la eficiencia con que éstas trabajan en conjunto les lleva a lograr metas comunes dependiendo básicamente, de las personas que dirijan la empresa. De ahí, la importancia del avance tecnológico y el desarrollo del conocimiento, cuando son pincelados con una acertada gerencia capaz de combinar destellos con el talento humano a su disposición.

Es aquí, donde está la relevancia de la actividad gerencial, pues el contexto predominante es riguroso y complicado, imponiendo desafíos donde el "deber ser" del gerente es saber qué está ocurriendo en su entorno, estableciendo enfoques facilitadores de comprender lo nuevo, y de la necesidad de un cambio hacia lo mejor, en este caso el abordaje en la nave de la tecnología, como herramienta para adentrarse en un mundo lleno de expectativas y conocimiento. 
En este sentido, es de suponer que las organizaciones del siglo XXI, esencialmente inundan a la generación de nuevas ideas, para ser más competitivos y lograr transformar a la sociedad, en una basada en el conocimiento. Para Malvicino y Serra (2006), "el conocimiento es el único recurso ilimitado, el único activo que aumenta con su uso" (pág. 86) bajo esta premisa, se está dando un gran cambio del prototipo sociedad industrial al paradigma de la sociedad del conocimiento, como se mencionó anteriormente.

De allí, la necesidad de evaluar el estilo gerencial presente en las organizaciones con miras a entender que deben gerenciar el discernimiento, en la búsqueda de formas que permitan crear un valor adicional mediante la captura, almacenamiento, comercialización del conocimiento, esto supone a la sociedad a sumergirse en el mar de las transformaciones, razón por la cual las instituciones avocadas a impartir instrucción deben según Soriano, (2000) "ser parte fundamental del desarrollo cultural, socioeconómico, tecnológico de los individuos, comunidades y naciones" (pág. 56).

Desde esta perspectiva, va dirigido el presente estudio titulado "Gerencia y Sociedad del Conocimiento para Optimizar el Desempeño Académico del Docente", donde se pretende lucir a la nueva gerencia como avanzada no por la tecnología, sino por la comprensión profunda de los cambios sociales, humanos, y las generaciones futuras. Así, esto esboza una nueva realidad para la gestión, una nueva identificación: una dirección que se autoconstruye en los abstractos caminos de la sociedad del conocimiento.

Amparados en esta visión, el objetivo que sustenta el estudio es "Identificar la importancia de la Gerencia y Sociedad del Conocimiento para Optimizar el Desempeño Académico del Docente", para ello se observa que el "deber ser" del docente en el siglo XXI, debe verse desde una óptica integradora, constructivista, relacional, siendo ante todo formador, innovador y creativo facilitador del desarrollo de todas las potencialidades humanas. 
Dentro de esta óptica, el desempeño académico del docente sería entonces asesor, facilitador del aprendizaje, o sea el creador de ambientes de aprendizaje, contextos, donde actúe como gerente de la información, conocimiento, recursos, espacios y tiempos, motivando en el momento necesario para lograr la transformación de la organización donde cumple su misión de multiplicador de aprendizajes.

El artículo está escrito debido a la inquietud de la realidad actual, en su proceso de ajuste y equilibrio ante los retos asumidos como constructores de la gerencia para la sociedad del conocimiento. Hoy día, se está pasando del modelo de la sociedad industrial característica de la modernidad, al paradigma de la sociedad del conocimiento como representación de la postmodernidad, de la mano con la globalización y las tecnologías de la información y comunicación (TIC). De acuerdo con lo anterior señalado, se enfatiza el uso de una metodología sustentada en la lectura de reconocidos autores; referencias de textos bibliográficos, para dar soporte al análisis teóricobibliográfico de las diversas fuentes que van a permitir determinar las características de la gerencia y la sociedad del conocimiento del estudio planteado.

\section{Gerencia}

La ventana que abre nuevas perspectivas a las organizaciones actuales, amerita de gerentes fortalecedores de los canales participativos para la intervención activa de los trabajadores, al momento que identifican prioridades y formulan objetivos, además de ejercer un adecuado control sobre la organización. Al respecto, Añez, (2005), establece el gerente "debe ser eficaz, promover la concertación interna y externa, haciendo compatibles los intereses en los distintos sectores $u$ organizaciones afines $y$ coordinar esfuerzos y recursos para el logro de los objetivos económicos y sociales de la empresa" (pág. 78). 
En otras palabras, en el gerente debe ser trascendental su actuación poseer inteligencia para alcanzar los objetivos propuestos, identificar y evaluar las oportunidades, amenazas provenientes del contexto en que está inmerso a la vez que las analiza, para la consecución de cambios basados en conocimientos de actualidad. Ante esta realidad, las transformaciones dentro de la organización serán lideradas por el gerente quien habrá de estar formado, capacitado y adiestrado debido a la alta responsabilidad asignada a su persona para enfrentar con victoria los nuevos modelos relacionados con los procesos gerenciales de la nueva cultura colectiva.

Ante las nuevas realidades en las organizaciones, según Hernández, (2002): establece "se ha de formar, capacitar y perfeccionar al personal orientados a ser sujetos claves para un óptimo desempeño, donde no es menos importante la formación gerencial...requeridos para desempeñar su rol y aflorar sus características personales" (pág. 32).

Sobre la base de las consideraciones anteriores, las circunstancias cambian tan inesperadamente que no nos damos cuenta. El gran cambio, es que vivimos en una sociedad regida por la información, el conocimiento y los avances tecnológicos, esto transforma nuestras vidas, la de las empresas y la gerencia. Entretanto, muchos gerentes siguen gerenciando sin reconocer que viven en una sociedad gobernada por la información, el conocimiento, la comunicación y las tecnologías. Estas grietas persisten, cuando en los espacios educativos no llegan a generar nuevos conceptos y teorías que nos expliquen las cosas en esta sociedad del conocimiento.

Al respecto, Rojas, (2006), al comunicar en su conferencia sobre los retos de la gerencia en la sociedad de la información establece "cada día es mayor la responsabilidad de dirigir, haciéndose mayor la necesidad de producir nuevos conocimientos propios para gerenciar en la era de la información. Dirigir sin educación, sin conocimientos avanzados puede ser un salto atrás" (pág. 81). Es decir, el compromiso del gerente hoy es de tal magnitud que es 
necesaria una nueva forma de educación o sea, una nueva forma de edificar organizaciones. Por ello, uno de los grandes desafíos de la gerencia ante esta nueva sociedad del conocimiento, es entender que estamos viviendo en la era informacional donde la manera de producir saberes, indagaciones, discernimientos y la forma de procesarlos, valorarlos es la llave de oro que nos abrirá las puertas de la fortuna y la prosperidad.

\subsection{Sociedad del Conocimiento}

\subsubsection{Conocimiento}

A fines del siglo XX y comienzos del siglo XXI florecen dos términos que se manejan actualmente: "Sociedad de la Información y Sociedad del Conocimiento". Bell, (1973), sociólogo estadounidense introdujo la noción de la «sociedad de información» en su libro: El advenimiento de la sociedad postindustrial, donde formula que el eje principal de ésta será el conocimiento teórico y advierte que los servicios basados en él habrían de convertirse en la estructura central de la nueva economía y de una sociedad apuntalada en la información, donde las ideologías resultarían sobrando.

A juicio de, Torres, (2005), esta expresión "reaparece con fuerza en los años 90, en el contexto del desarrollo de Internet y de las TIC" (pág. 18). El término en inglés de la Sociedad del Conocimiento "Knowledge Society" emergió hacia finales de los años 90; es empleada particularmente, en medios académicos, como alternativa que ciertos prefieren a "sociedad de la información".

En este sentido, la Organización de las Naciones Unidas para la Ciencia, la Educación y la Cultura, UNESCO (ob.cit), ha adoptado el término "sociedad del conocimiento", o su variante "sociedades del saber", dentro de sus políticas institucionales, para lo cual ha desarrollado una reflexión en torno al tema, que busca incorporar una concepción más integral, no en relación únicamente con la dimensión económica, sino también en el orden social, 
cultural y académico. Es por ello, que las instituciones educativas se ven aludidas de manera directa, por ser éstas las gestoras naturales del conocimiento, tienen el compromiso de satisfacer nuevos mandatos de la sociedad que las sustenta, la investigación y la extensión de carácter pertinente, social, pero sobre todo con la masificación de la cultura y los saberes, sin perder de vista la calidad y eficiencia que impone la sociedad del conocimiento.

Asimismo, Malvicino y Serra (ob.cit), exponen "el conocimiento es el único recurso ilimitado, el único activo que aumenta con su uso" (pág. 2), bajo esta premisa, se está dando un gran cambio del paradigma sociedad industrial al paradigma de la sociedad del conocimiento. De allí, la necesidad de evaluar el estilo gerencial presente en las organizaciones con miras a entender que deben gerenciarlo bien, en la búsqueda de formas que permitan crear un valor adicional mediante la captura, almacenamiento y comercialización de éste.

Sin embargo, a criterio de Senge, (2005) "la información no es lo mismo que el conocimiento" (pág. 48) ya que, la información es ciertamente una herramienta del conocimiento, pero no es éste en sí, sustentando el autor que la única manera de sobrevivir a los cambios exigidos por el entorno es construyendo organizaciones perspicaces que permitan manejar nuevos modelos mentales, aceptando la apertura a nuevos paradigmas del tipo organizacional, gerencial y tecnológico. Tal es el caso, de la incorporación sistemática de las Tecnologías de Información y Comunicación (TIC) en la sociedad moderna, porque está asumiendo consecuencias drásticas, al punto de provocar cambios estructurales en el orden político, cultural, educativo y social.

De todo este proceso aplicado, se sugiere que la formación del futuro docente enclaustre el apoderarse de saberes, tendentes a gestar el conocimiento más que a transmitirlo como se ha venido planteando. Sin duda, estamos ante el reto de convertir estos saberes en discernimiento explícito, 
funcional, evidenciando su aplicación; exigiendo sumar elementos para su formación, que sitúe el acento en procesos de cambio e innovación, para un hacer crítico y reflexivo capaz de admitir y manifestarse más allá de lo operativo e instrumental y sea capaz de incluir en su praxis pedagógica el uso apropiado y conveniente de las TIC.

\subsection{Desempeño Académico del Docente}

Una de las urgencias que demanda la sociedad, apunta hacia la formación del desempeño académico del docente, con clara visión de las exigencias presentadas en el campo social, científico, humanístico y tecnológico, sólo con un docente de este tipo, se pueden encarar satisfactoriamente las demandas de una situación dinámica, cambiante, preparando al nuevo republicano para la transformación de la sociedad y por ende de su país. Díaz, (2004) sostiene lo siguiente:

El docente debe estar bien preparado en relación a su desempeño... y ello implica, no sólo la responsabilidad de transmitir conocimientos básicos, sino también el compromiso de afianzar en los educandos valores y actitudes necesarios para que puedan vivir y desarrollar sus potencialidades plenamente, mejorar su calidad de vida, tomar decisiones fundamentales y continuar aprendiendo (pág. 10).

Lo que quiere decir, ser docente es tener la oportunidad de enfrentarse cada día a una caja de maravillas colmada de: una sonrisa, un suspiro, un logro, un secreto difícil de responder, situaciones que hacen del ejercicio académico un rol gratificante y un reto permanente.

Amparados en esta visión, los docentes ante esta situación se enfrentan con la necesidad de romper con las estructuras o modelos de enseñanza ya establecidos desde épocas como estudiantes, cuando todo era memorístico y sólo transmisión de conocimientos, para darle paso a la escuela que enseña a aprender, donde el docente esté preparado desde el punto de vista técnico y 
pedagógico a la incorporación de nuevas tecnologías en los espacios educativos, esto conlleva a rediseñar y adaptar estrategias de enseñanza para promover aprendizajes significativos agregando nuevas tecnologías en su caudal de experiencias y vivencias.

Lo anterior se aúna a, no tratar de reducir la función del desempeño académico del docente a un simple situar o enganchar la información, sino de plantearse y cuestionarse ¿cómo integrar herramientas telemáticas a su práctica pedagógica?, ¿cómo diseñar, guiar o evaluar el proceso de aprendizaje realizado con el soporte de las TIC? Sin duda, todo esto requiere cambios en el enfoque de la formación del desempeño docente que va más allá de la nueva capacitación instrumental elemental para el manejo de las TIC; demanda el conocimiento de lenguajes visuales, flexibilidad, autonomía y compromiso con su hacer diario y accionar pedagógico. De ahí, se aprecie al docente como ese profesional altamente profesional y fértil, ligado a una evaluación del desempeño enmarcada en parámetros de eficiencia y calidad.

Ello implica, situarnos en una sociedad que demanda cambios, donde lo valioso es el capital humano para dar calor al frío de estas innovaciones propias de avances científicos y tecnológicos. Al respecto, Mertens, (2004), plantea "la búsqueda del equilibrio en la formación del futuro docente: por un lado, ajustarse a las necesidades sociales, personales, empresariales, tecnológicas y por otro, el designio de la realidad contemporánea global" (pág. 100).

De hecho, no implica apartarnos del ser humano, de sus intereses, su sentir; por el contrario, es acercarnos a él mediante acciones de respeto y convivencia en escenarios diferidos en tiempo. Este planteamiento, invita a adentrarnos a la realidad subjetiva e intersubjetiva para comprender la sociedad del paradigma tecnológico signado por la producción cultural que circula por las redes sociales. 


\section{Metodología}

La metodología, es una técnica de la investigación científica. En tal sentido, Bavaresco, (2001), determina "es la fase mecánica a penetrar en el manejo de métodos y técnicas... en especial documental o bibliografía, llevan al investigador a emplear los aspectos metodológicos, debiendo explicar cómo tendría que hacer en esa investigación en particular" (pág. 67).

Razón por la cual, se afirma que el presente estudio, es de carácter documental y descriptivo, por lo que los datos se recabaron por medio de fuentes directas e indirectas, como; documentos de diversa índole, elaborados y procesados con anterioridad al trabajo. Por su parte, Sabino, (2005), expresa que "la investigación documental constituye un procedimiento científico sistemático, de indagación, recolección, organización, interpretación y presentación de datos e información alrededor de una estrategia de análisis de documentos" (pág. 37). Entretanto, Chávez (2001), expresa que "las investigaciones descriptivas, son todas aquellas que se orientan a recolectar informaciones relacionadas con el estado real de las personas, objetos, situaciones o fenómenos, tal cual como se presentaron en el momento de su recolección" (pág. 34).

En cuanto al diseño de la investigación, Palella y Martins (2004): describen que el diseño se refiere "a la estrategia que adopta el investigador para responder al problema, dificultad o inconveniente planteado en el estudio". Para fines didácticos, se clasifican en diseño experimental, no experimental y bibliográfico.

\section{Consideraciones finales}

Las conclusiones a las que hemos llegado, luego de hacer una revisión exhaustiva de fuentes relacionadas con la temática expuesta, sirvieron para adquirir una visión más profunda del problema objeto de estudio y ofrecer saberes para la gerencia y sociedad del conocimiento, enfocados en el 
desempeño académico del docente, como la manera de incentivarlo para que la enseñanza transmita sea acorde a los cambios impuestos hoy por la nueva sociedad denominada: "Sociedad del Conocimiento".

Se podría resumir la idea de la Gerencia como: la síntesis del proceso enfocado para dar nuevas respuestas a las nuevas realidades, donde existe la obtención de saberes, centrada en la información y el conocimiento. La nueva gerencia se enfrenta a un cambio de sentido de aprendizaje, es decir para que ésta pueda generar más bienestar que beneficios debe repartir, reproducir y hacer crecer la riqueza de este tiempo, es decir la información, el conocimiento y la tecnología.

Se debe estar claro, lo primero que está cambiando es la sociedad, puesto que se refleja en ella la información, conocimiento, es una colectividad tecnificada, globalizada, competitiva, múltiple, y compleja de comprender. Estamos ante un paisaje provocador de retos, en los que lo fundamental radica en interpretar el papel que juega la creación del conocimiento en la búsqueda de alternativas que fortalezcan a los países para alcanzar su desarrollo económico, social y cultural.

Es indudable, que la educación representa un factor crucial en esta transformación social, en virtud de permitir la formación adecuada en el desempeño académico en el ser humano, donde prevalezca la capacidad crítica y creativa para interpretar la información y traducirla en conocimiento.

En este contexto global, rubricado por el valor otorgado al conocimiento como factor crucial para lograr transformaciones sociales, en pro de una mejor calidad de vida de los ciudadanos que transitamos en este siglo XXI, le corresponde un reto decisivo a los constructores de sociedad, los bien llamados "docentes". 


\section{Referencias}

Bavaresco, A. (2001). Proceso metodológico en la investigación (Cómo hacer un diseño de investigación). Ediluz. Maracaibo.

Bell, D. (1973). Haunting the Knowledge Economy. New York: Basic Books. Chávez, N. (2001). Introducción a la Investigación Educativa. 1era. Edición. Zulia, Venezuela.

Díaz, M. (2004). Ensayo sobre Ética, Conocimiento y Gerencia. s.e. Hernández, R. (2002). Determinación del Gerente de Recursos Humanos de las Empresas que le presta servicios a la Industria Petrolera. Formación Gerencial, Año 1, Nro 1, LUZ. Núcleo Costa Oriental del Lago. Venezuela.

Malvicino, S. y Serra, R. (2006). La Importancia de la Gestión del Conocimiento y el Desarrollo del Capital Humano en las Organizaciones del Siglo XXI. Gestión del Conocimiento, 2.

Mertens, L. (2004). Competencia laboral: sistemas, surgimiento y modelos. Montevideo: CINTERFOR/OIT. Recuperado el 22 de Mayo de 2016 del sitio Web del Centro Interamericano para el Desarrollo del Conocimiento en la Formación Profesional (OIT/Cinterfor).

Organización de las Naciones Unidas para la Ciencia, la Educación y Cultura (2009). Sociedad del conocimiento. UNESCO.

Palella, S. y Martins, F. (2004). Metodología de la Investigación Cuantitativa. Caracas: FEDUPEL.

Rojas, L. (noviembre 2006). Los Retos de la Gerencia en la Sociedad de la Información. Conferencia Magistral correspondiente a la Clase Inaugural del Doctorado en Gerencia Avanzada 2da Cohorte Universidad Fermín Toro Septiembre 22 de 2006 NEGOTIUM / Ciencias Gerenciales Año 2 / Nro 5 / Los Retos de la Gerencia en la Sociedad de la Información 77-100. 
Sabino, C. (2005) Metodología, diseño y desarrollo del proceso de investigación. Colombia: McGraw-Hill.

Senge, P. (2005). La Quinta Disciplina. El arte y la práctica de la organización abierta al aprendizaje. Ediciones Granica. Buenos Aires.

Soriano, M. (2000). El Profesor Universitario ante los Retos del Mundo de hoy: sus competencias laborales. Instituto Superior Politécnico José Antonio Echeverría. La Habana, Cuba.

Useche, M. y Queipo, B. (2005). Las Organizaciones Posmodernas en el siglo XXI. Mañongo, Nro 24, 207-216. 


\section{María Mercedes Carrillo}

e-mail: mercecar-42-68@hotmail.com

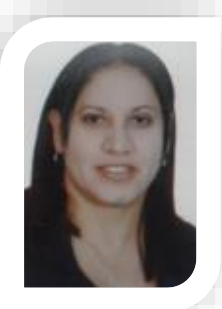

Nacida en Monte Carmelo, Estado Trujillo. Actualmente, cursa estudios de Doctorado en Ciencias de la Educación

en la Universidad Nacional Experimental "Rafael María Baralt". Magister Scientiarum en Administración de la Educación Básica. Licenciada en Educación Integral. Participante en el año 2010 de Actualización del Docente (300 horas). Ponente y participante del 1er y 2do Congreso Pedagógico en la Escuela Bolivariana "Antonio Nicolás Briceño", municipio Motatán, estado Trujillo. Experiencia Laboral actualmente docente de aula en la EPB "Antonio Nicolás Briceño". 